\title{
Attitudes Toward Vehicle-Based Sensing and Recording
}

\author{
Manya Sleeper*, Sebastian Schnorf $\nmid$, Brian Kemler $\ddagger$, Sunny Consolvo $\ddagger$
}

\author{
*Carnegie Mellon University \\ Pittsburgh, PA, USA \\ msleeper@cmu.edu
}

\author{
$\dagger$ Google \\ Zurich, Switzerland \\ sebschnorf@google.com
}

\author{
$\ddagger$ Google \\ Mountain View, CA, USA \\ \{bkemler, sconsolvo\}@google.com
}

\begin{abstract}
Vehicles increasingly include features that rely on hi-tech sensors and recording; however, little is known of public attitudes toward such recording. We use two studies, an online survey $(n=349)$ and an interview-based study $(n=15)$, to examine perceptions of vehicle-based sensing and recording. We focus on: 1) how vehicle-based recording and sensing may differ from perceptions of current recording; 2) factors that impact comfort with vehicle-based recording for hypothetical drivers versus bystanders; and 3) perceptions of potential privacy-preserving techniques. We find that vehiclebased recording challenges current mental models of recording awareness. Comfort tends to depend on perceived benefits, which can vary by stakeholder type. Perceived privacy in spaces near cars can also impact comfort and reflect mental models of private spaces as well as the range of potentially sensitive activities people perform in and near cars. Privacypreserving techniques may increase perceived comfort but may require addressing trust and usability issues.
\end{abstract}

\section{Author Keywords}

vehicles; cars; sensors; recording; cameras; perceived benefits; attitudes; privacy; privacy-preserving mechanisms

\section{ACM Classification Keywords}

H.5.m. Information Interfaces and Presentation (e.g. HCI): Miscellaneous

\section{INTRODUCTION}

Vehicles increasingly include "smart" features that rely on hitech sensors and recording, such as back-up cameras, traffic detection, automatic lane correction, object detection, location and behavior tracking, as well as adaptive braking and cruise control $[25,20]$. These "smart" features rely on sensors and recording including external video and audio, lidar (laser-based radar), radar, and GPS.

Permission to make digital or hard copies of part or all of this work for personal or classroom use is granted without fee provided that copies are not made or distributed for profit or commercial advantage and that copies bear this notice and the full citation on the first page. Copyrights for third-party components of this work must be honored. For all other uses, contact the owner/author(s). Copyright is held by the owner/author(s)

UbiComp '15, September 7-11, 2015, Osaka, Japan

ACM 978-1-450303574-4/15/09.

http://dx.doi.org/10.1145/2750858.2806064
This vehicle-based sensing and recording can bring recording into increasingly ubiquitous spaces, adding recording to publicly visible areas that may currently be considered private or unrecorded. Many of the features, like external cameras, or radar, also capture areas around vehicles. They may sense or record varied indirect stakeholders, other than the driver of the vehicle, including passengers, drivers of nearby vehicles, pedestrians, people at their homes, etc. Unlike the driver, indirect stakeholders (referred to in this paper as bystanders) may have less perception of or control over the recording.

Little is currently known about public attitudes and sensitivities toward emerging vehicle-based recording. However, as such recording becomes more ubiquitous, it is important to understand how it may be perceived, including how perceptions may vary for bystanders, to whom benefits may accrue differently. This understanding can help designers develop features and education to promote all stakeholders' comfort as vehicle-based recording becomes more common.

We conducted two studies to explore perceptions of vehiclebased recording and sensing. We performed an online survey to explore perceived benefits and sensitivities to being recorded by cars in three hypothetical scenarios: 1) as the driver of a "smart" car; 2) as a driver near a "smart" car; and 3) when generally recorded (e.g., as a non-driver). In a follow-up experience-sampling and interview-based study we examined perceptions of current recording during everyday outdoor and transportation activities, comfort with vehiclebased recording, and reactions to three potential privacypreserving techniques. We focus on three research questions:

1. How does vehicle-based recording and sensing align with current perceptions of recording and mental models of awareness? How might it be unexpected?

2. What factors impact perceived comfort with vehicle-based recording and sensing? How do these factors vary by potential stakeholder type?

3. How are potential privacy-preserving techniques that could be applied to car-based recording/sensing perceived? What issues might need to be addressed in application?

We find that, at least in the near-term, car-based recording and sensing may be unexpected. Such recording may occur in spaces where participants feel unrecorded and may challenge current mental models for perceiving recording. We also find that perceived comfort with car-based recording and sensing tended to vary based on perceived benefits, especially related 
to driving. However, those perceived benefits varied by hypothetical stakeholder group. In general bystander scenarios, respondents tended to describe general public safety instead of driving-related benefits. Comfort also varied based on perceptions of publicly visible locations in and around vehicles as private spaces, which created a dynamic of semi-private spaces. In such spaces respondents described varied sensitive activities they preferred not to have recorded. In light of these sensitivities, participants tended to feel more comfortable with privacy-preserving mechanisms, but suggested some usability and trust-related challenges in application.

\section{RELATED WORK}

Technologies with recording and sensing such as wearable devices, CCTV (closed circuit television cameras), and smart home systems bring recording into varied spaces. Vehicles are poised to add additional recording capacity, with increasing recording and sensing-reliant features, in potentially unexpected locations. This will prompt new dynamics, creating a need to explore how perceptions of, and comfort with, such recording varies from previously-studied scenarios.

\section{Perceptions of recording and ubiquitous technology}

Many technologies include recording and sensing, including home sensing and recording systems $[9,18,24]$, personal and wearable devices ranging from mobile phones and firstperson video devices to personal healthcare devices [22, 26, 28], as well as CCTV and other infrastructure-based cameras. The increasing ubiquity of such technologies can lead to recording and sensing in varied spaces.

Perception of, and comfort with, such recording varies. General perceptions tend to draw on heuristics including whether locations are private, public, or shared (e.g., office spaces), as well as past experience and visual cues like signs and cameras [17]. Comfort tends to vary based on individual and contextual factors. For general video recording, privacy concerns can depend on perceived privacy risks, including the four risks included in the Concern for Information Privacy Model: collection, access, secondary use, and errors. Comfort also varies based on perceived benefits from the recording and ability to consent [21]. For public spaces comfort can also depend on individual traits such as gender and psychological well-being and contextual factors including social expectations and the location and scale of the observer [12].

In home environments, comfort with sensing and recording similarly tends to depend on perceived benefits, with an additional focus on potentially embarrassing or sensitive activities that may occur in the privacy of the home. Choe et al. found, for example, that participants provided with in-situ prototypes of in-home sensing devices perceived benefits, especially related to safety or monetary gain. However, they also perceived downsides, including general discomfort with monitoring and concerns about sensitive activities being captured [9]. In additional work, participants identified a range of potentially sensitive or embarrassing activities that they would not want recorded by home systems [8, 24].

Comfort with recording and sensing can also vary based on whether people are primary users of technologies like first- person recording devices or bystanders. Bystanders can have additional concerns about identifiability, purpose of recording, perceptions of the recorder, and location privacy [11, 22]. In a study of perceptions of a personal audio device [3], Iachello et al. also found that bystanders can have concerns about ability to consent to the recording and unauthorized follow-up use of the content [13].

\section{Vehicle-based recording and sensing}

Vehicles increasingly include features that rely on hi-tech sensors and recording (referred to in this paper as recording). These features include assistive technologies such as object detection, automatic lane adjustment, parking assistance, and automatic braking and cruise control. They also include features like directions and traffic detection that can rely on vehicle-based sensors and a centralized server. These features use sensors including external cameras, radar, lidar, and GPS [19]. Services also provide tracking for safety or directions [2]. Insurance companies also provide devices to track car location and driver behavior, sometimes with incentives [20,31]. Additionally, vehicles increasingly include event data recorders, which serve as "black box" devices and record a sliding window of data about the vehicle that can be used to assess the cause of accidents [30].

In this paper we refer to vehicles that include such hi-tech recording as "smart" vehicles and focus on several features: external cameras, GPS, radar, and event data recording. We chose these features because they are in use, provide varied benefits, and capture data that could be considered sensitive.

Vehicles are often ubiquitous, present in public and semiprivate spaces (e.g., roads, near homes, etc.), mobile, and may record in situations that may not match current mental models for determining when recording is present. The range of stakeholders who may be impacted by such recording, including drivers and varied bystanders, may also complicate perceptions of such recording. Although many hi-tech recording features are currently limited to a relatively small number of higher-end vehicles, cars increasingly include such features [16]. Thus, we address a scenario that assumes the relatively ubiquitous presence of such capabilities.

We examine perceptions of vehicle-based recording to inform the development of such technologies in a manner sensitive to public perception. As vehicle-based recording becomes more ubiquitous it is important to understand how factors that impact comfort may differ from other modalities, as well as how factors may vary for different stakeholders.

\section{Privacy-preserving mechanisms}

Technical mechanisms can be used to reduce the sensitivity of recording, which also could be applied to vehicles. However, such mechanisms must be contextually trustworthy and usable. We focus on three privacy-preserving mechanisms: local data storage (local storage), blurring personally identifiable information in images (blurring PII), and providing location-defined privacy controls (geo-fencing).

Blurring, or otherwise removing identifiable information, can be used to protect privacy in video recordings or photos. 
Google Street View, for example, blurs faces and license plates as a privacy-preserving technique [1]. However, to be effective, blurring must balance the effectiveness of the privacy protection with the usefulness of the data [29]. Local storage presents another option for increasing trust or preserving privacy. This technique can be used when data can either be sent to a centralized system or processed locally, in a device controlled by the user. Trust tends to increase for local processing, although mental models can vary [14].

Data access can be controlled at a number of levels for ubiquitous technologies. One option is to use geo-fencing to allow users to set privacy rules according to geographic boundaries. In cars, for example, some companies allow parents to set boundaries to track their children. Developing effective geo-fencing controls can be challenging, however, because options must align with contextually-specific factors [32].

Applying privacy-preserving mechanisms requires contextually-specific education, notification, and control. We offer an initial discussion of potential considerations associated with these mechanisms for car-based recording.

\section{METHODS}

We use two studies to explore attitudes toward vehicle-based recording. Study 1 is an online survey of US drivers focused on perceptions of potential benefits and privacy sensitivities related to being recorded by "smart" cars in different scenarios $(\mathrm{n}=349)$. In Study 2, a follow-up experience-sampling and interview-based study of US and Swiss participants $(n=15)$, we focused on exploring how perceived comfort with potential car-based recording/sensing varied based on everyday activities and potential privacy-preserving techniques.

\section{Study 1: Survey of varied scenarios}

We first explored high-level attitudes toward "smart"-carbased recording. We focused on perceived benefits and privacy sensitivities in three hypothetical stakeholder scenarios: (1) driving a "smart" car with sensing and recording functionality; (2) driving near such a "smart" car; and (3) recorded as a general bystander.

\section{Survey}

We performed a survey of 349 US Amazon Mechanical Turk workers (MTurkers), who reported driving at least monthly, in August 2014. We paid \$0.50.

In prior work Choe et al. explored privacy sensitivities for home recording by examining home activities people would not want recorded [8]. We drew from this structure. However, vehicles differ from the home. They are often present in spaces that include people with varied perceptions of the benefits from, and control over, the recording. For example, a "smart" car on the street might record data about not only the car's occupants but also pedestrians, other drivers, bikers, and people in the windows of buildings. We, therefore, asked respondents for reactions to a hypothetical "smart" car that recorded GPS, external video, and radar data for safety purposes, imagining that they were in one of three randomlyassigned stakeholder conditions:

- the driver of the "smart" car (n=140) (denoted as "D")

\section{- driving near a "smart" car (n=87) ("DN")}

- a general bystander (not necessarily driving) recorded by a "smart" car (n=122) ("B")

The conditions represent varied potential stakeholders. Each group may perceive different benefits and downsides, based on their relationship to the car performing recording. For example, the driver of a "smart" car might perceive direct driving benefits but also risks (e.g., perceived help with parking but fear of speeding being detected). Alternatively a driver near a "smart" car might perceive driving-related risks but only indirect driving-related benefits. For example, they would not get help with their own parking, but might benefit from someone else's improved parking, and they still might fear having a nearby car detect their speed.

After being introduced to the condition, respondents answered three sets of free-response questions. We began with two targeted warm-up questions to prompt respondents to think about condition-related behaviors [6]. In the driving conditions we asked them to think back over the previous month and describe three ways they used their car and three ways they benefited from driving. In the bystander condition they described three (non-driving) activities and locations during/in which they could see cars or other vehicles.

Next, we asked two questions related to perceived benefits and two questions related to perceived sensitivities. One focused on habitual activities during which they felt they would benefit from, or be sensitive to, car-based recording/sensing: "Please describe a situation that happens to you regularly [WHILE DRIVING] that you wouldn't want captured by these systems" and "Please describe a situation that happens to you regularly [WHILE DRIVING] that you think would benefit from these cars having these systems." The other focused on more extreme events that may occur occasionally: "Please describe the one situation in the last year that you would have been most upset to have had captured by these systems" and "Please describe the one situation in the last year [THAT TOOK PLACE WHILE YOU WERE DRIVING] during which you would have been happiest to [HAVE HAD THESE SYSTEMS/THAT CARS HAD THESE SYSTEMS]." Question wording varied slightly to fit each condition (variation indicated in bracketed text). Respondents also provided demographics and familiarity with hi-tech car features.

\section{Survey analysis}

One researcher iteratively created four codebooks for the perceived benefits and sensitivities. We based the codes for the perceived sensitivities on codes used by Choe et al. [8].

\section{Study 2: Comfort during everyday activities}

We also wanted to probe current perceptions of recording during everyday activities, how comfort with car-based recording varies, as well as perceptions of potential privacy-preserving techniques. We performed a follow-up, two-part, experiencesampling-grounded interview-based study with US and Swiss participants ( $\mathrm{n}=15,6$ US and 9 Swiss) in September 2014. In Study 2 we included both US and Swiss participants in an effort to include participants with two potentially different cultural and legal perspectives on public recording. 


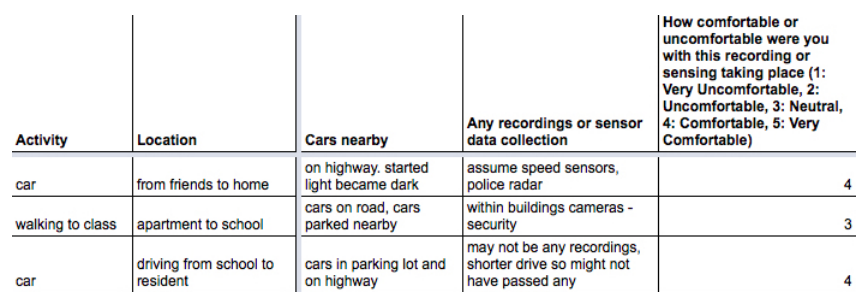

Figure 1. Study 2 interviews were grounded around sheets created from participants' reported outdoor and transport activities. This figure provides an example of a portion of one of the sheets. The full sheets included additional study questions as well as the date, time, and type of the activity.

We grounded the interviews around participants' everyday activities that could potentially be captured by ubiquitous vehicle-based recording. We recorded the activities during a seven-day, pre-interview, experience-sampling period. We then used the activities to explore perceptions of, and attitudes toward, current perceived recording/sensing (referred to collectively for the remainder of the paper as recording) during the activities as well as potential "smart"-car-based recording during the activities under several conditions.

\section{Outdoor and transport activities reported}

Participants reported outdoor and transportation activities for seven days. We focused on these activities because, in piloting, they covered many activities and locations that may be captured by ubiquitous vehicle-based recording.

Participants received instructional emails and installed Paco, an experience-sampling application, on their phones. ${ }^{1}$ They received five "pings" (alerts) at random times each day. With each they were prompted to fill out a short survey about recent or current outdoor (e.g., walking, eating outdoors, etc.) or transportation (e.g., driving, biking, etc.) activities, if such activities had occurred. They described: 1) What they were doing (e.g., type of activity or transport); 2) Where they were; and 3) Whether there were cars or other vehicles nearby.

Participants who completed at least 12 experience-sampling surveys qualified for the final interview (17 total). Fifteen participants are reported; one was removed due to a technical malfunction, and the other dropped out.

\section{Reported activities used to ground interview}

We used the activities from the pre-interview responses to create discussion sheets for each participant's interview (Figure 1). Collecting short descriptions of the activities in-situ, and then following up with an interview-based discussion allowed us to ground the discussion in participant's everyday activities while minimally biasing the activities [10]. We interviewed participants immediately following data collection, and participants were able to easily remember the activities. Each sheet typically consisted of up to 15 of the recorded transportation and outdoor activities. If a participant submitted more than 15 activities we selected a set of 15 that represented a range of activities and locations (for three participants, P07, P12, and P15, we included one or two extra items to fully represent the range of activities and locations

\footnotetext{
${ }^{1}$ https://www.pacoapp.com
}

reported). The sheet also consisted of a column for each interview question, which was hidden and then revealed and filled in for discussion during the interview. This general structure drew on prior work on general perceptions of recording [17].

\section{Interview on perceptions and attitudes toward recording}

We used the discussion sheets to ground hour-long, semistructured, interviews during which we:

- Discussed each activity to refresh participants' memories

- Asked participants to describe any recording they thought currently took place during each activity and rate how comfortable or uncomfortable they were with it

- Described three types of "smart"-car-based recording: external cameras, radar, and electronic data recorders. We asked participants for reactions to the technologies and to describe any benefits or downsides for each. If the participant could not provide any benefits or downsides, we provided "Devil's advocate" arguments for the other side to prompt two-sided discussion (based on the interview technique used in Choe et al. to introduce home recording [9])

- Asked participants to rate how comfortable or uncomfortable they would be with these types of technologies recording them for each activity assuming the data was shared with a "service provider"

- Asked participants about how their comfort would vary for three potential privacy-preserving techniques: if data was only stored in the car (i.e., not shared with a service provider), if personally identifiable information was blurred, or if they could set certain areas to private for which data would not be sent to a service provider

Most interviews (9) were done remotely using screen sharing. Six interviews in Zurich, Switzerland were performed in person. Audio recordings were made and transcripts created.

\section{Analysis}

We looked at factors participants felt would impact their comfort with recording. The interviewer created an initial set of codes around factors that impacted comfort, and trends in perceived impacts of privacy-preserving mechanisms, by creating affinity diagrams from her notes. She then coded the interview transcripts, iteratively updating the codebooks. The trends from the final codebooks are described.

\section{Study limitations}

Reported attitudes may not match behavior in practice [23]. Consideration of emerging technologies also often requires reactions to hypothetical scenarios. We, therefore, sought to ground discussions in participants' actual activities, to minimize reactions to hypothetical aspects of technology. We combined two approaches to balance some biases of each. We grounded Study 1 in reactions to past events. However, asking for reactions to only benefits or downsides, rather than allowing consideration of tradeoffs may have prompted more extreme responses. We used a more discussion-based approach in Study 2 and grounded the study in multiple activities to allow participants to express tradeoffs. However, we used a 


\begin{tabular}{l|lllll} 
Code & Country & Gender & Age & Occupation & Children \\
\hline P01 & Swiss & F & $31-40$ & Researcher & y \\
P02 & USA & M & $18-23$ & Student (chem. eng.) & n \\
P03 & Swiss & M & $41-50$ & Unemployed & n \\
P04 & Swiss & M & $41-50$ & Finance director & y \\
P05 & Swiss & F & $31-40$ & Stay-at-home parent & y \\
P06 & Swiss & M & $51-60$ & Chef & n \\
P07 & USA & F & $18-23$ & Service worker & n \\
P08 & USA & F & $18-23$ & Nanny & n \\
P09 & Swiss & M & $18-23$ & Financial research assoc. & n \\
P10 & Swiss & F & $24-30$ & Student (geophysics) & n \\
P11 & USA & M & $31-40$ & Animal boarding specialist & y \\
P12 & USA & M & $51-60$ & Retired & n \\
P14 & Swiss & F & $51-60$ & School tech. coordinator & y \\
P13 & Swiss & M & $24-30$ & Student (comp. chem.) & $\mathrm{n}$ \\
P14 & USA & F & $24-30$ & Culinary student & $\mathrm{n}$ \\
P15 & USA & M & $24-30$ & Candy shop manager & $\mathrm{n}$ \\
\hline
\end{tabular}

Table 1. Study 2 participant demographics.

Devil's Advocate approach to ensure that respondents who were unable to independently volunteer pros and cons considered a range of scenarios when we initially presented the technologies. While this approach may have biased participants, we only used it, sparingly, in the initial introduction.

\section{RECRUITMENT AND DEMOGRAPHICS}

\section{Study 1 recruitment and demographics}

Study 1 respondents included 349 US MTurk workers who drive a car at least monthly (69\% reported driving daily or more). Respondents skewed slightly young (17\% 18-23, $40 \%$ $24-39,27 \% 31-40,17 \%$ over 40 ), but included a range of ages, mix of genders, and variety of occupations (55\% male; $16 \%$ students, $18 \%$ work in IT).

Respondents reported some familiarity with hi-tech car features that often include recording. Most respondents had used GPS/satellite navigation (80\%). Most were familiar with, but had not used, features that rely on external cameras such as parking assistance (70\% were familiar, 13\% had used), backup cameras $(57 \%, 35 \%$ had used), and automatic lane correction $(51 \%, 13 \%$ had used).

For Study 1 we used an MTurk survey to achieve the desired sample in a time and cost-effective manner. The MTurk worker pool has been found to provide acceptable demographic diversity and performance compared to other sources $[5,7]$. However, it has some known demographic biases. US MTurkers tend to be trend younger, more female, and lower income than the US population [15]; compared to other online sample sources they also tend to skew more privacy sensitive [27].

For quality control we also limited the survey to MTurkers with at least a 95\% approval rate on the site. MTurk uses worker approval ratings to allow requestors to filter out MTurkers who regularly perform lower-quality work, for example completing studies with nonsensical entries.

\section{Study 2 recruitment and demographics}

For Study 2 we used a recruiting service (Table 1). We recruited for a mix of ages (over 18), genders, occupations,

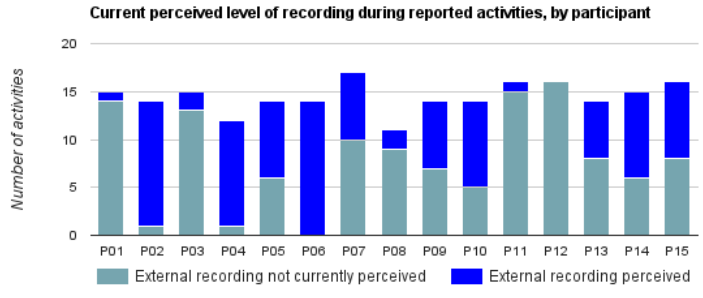

Figure 2. In Study 2, current perceived levels of external recording during reported activities varied by participant.

and tech-savviness. To compare participants with and without children, we recruited some participants with children under 18. We also hoped to see if different privacy-related cultural and legal environments might impact attitudes, so we recruited US and Swiss respondents for comparison. In our sample this did not emerge as a distinctive factor, so we do not report country-specific results. We also screened for participants who reported varied sensitivities to CCTV cameras to try to include participants with nuanced views, and for participants who used smartphones so that they could use Paco.

\section{RESULTS}

Vehicle-based recording brings recording capacity into potentially unexpected spaces to capture varied stakeholders. Respondents had varied baseline expectations of recording in spaces that may be captured by ubiquitous vehicle-based recording. Perceived comfort with potential car-based recording tended to vary based on perceived benefits, conceptions of privacy levels in the semi-private spaces in and near vehicles, as well as conceptions of collection, access and identifiability. Privacy-preserving mechanisms were generally perceived positively, especially for addressing access and identifiability, but participants also expressed some distrust or feelings that the mechanisms may not meet their needs in application.

We describe these themes, drawing on data from both studies. Study 1 quotes are denoted as "D," "DN," or "B" based on respective condition (driver, driving near, or bystander), while quotes from Study 2 are denoted by participant number.

\section{Car-based recording outside baseline expectations}

Outdoor and transportation activities present opportunities for vehicle-based recording. Current perceptions of recording varied for reported activities, and vehicle-based recording may challenge current experiences and mental models that shape expectations for such spaces.

In Study 2 we discussed 217 activities, reported via Paco, during which vehicle-based recording could potentially occur, across the interviews. Participants provided 78 outdoor activities including eating, exercising, yard work, house chores, shopping, playing or relaxing, recreational walking, and working. They also reported 139 transportation activities including driving, getting rides, public transportation, biking, walking, and, in one case, using a scooter. During most activities cars were nearby (e.g., parked, driving past, etc.).

We probed baseline perception of current recording by asking when participants thought "recordings were being made 
or sensor data was being collected" for each activity. Participants perceived current recording during $60 \%$ of the activities. They described data collection from their own smartphones or GPS, video security cameras, typically in stores, banks or on public transportation, and traffic or speed cameras when on or near roads. However, perception of external recording, from devices participants did not control (i.e., not their own phones or cameras), varied (Figure 2).

Some participants (e.g., P01, P03, P11, P12) perceived minimal external recording, while others perceived external recording during most activities (e.g., P02, P06). Other participants had nuanced expectations based on factors similar to those in prior work, including norms, heuristics, and visual cues [17]. They tended to feel unrecorded around homes, and thought recording was more likely in public spaces. P08 described: "I don't work in, like, an office or something. I work in a house so there isn't really recording devices here." Similarly, participants assumed less recording in less populated areas, for example, "Possibly not in the bus...it's quite a small bus...just goes around the village" (P05).

Participants also sometimes drew on visual cues including visible cameras or signs, for example, "...on the trains there are signs like this car is being monitored" (P13). Some participants drew on past experience; P14 assumed there were cameras on the train "because a while back there was, like, a story on the news where they showed this, uh, this guy, like, shooting someone on the [anonymized bus line] or something crazy like that, and they captured it."

Thus, there were varying gaps between current expectations of recording and potential vehicle-based recording that could occur during transportation or in outdoor spaces. Some heuristics used to judge whether recording was taking place may fall short for vehicle-based recording. For example, vehicles are often present near homes and in spaces not commonly viewed as public, which may not be perceived as recorded. Vehicles also can be present in less populated areas, for example, on large highways near small towns.

Cars also tend not to have visible cues, such as signs or visible cameras, to indicate recording. Accordingly, most respondents described, when asked, not knowing how to recognize a vehicle with recording capabilities. The most common heuristic provided was a "new" or "expensive" car. As vehicle-based recording becomes increasingly ubiquitous, this dynamic could lead to uncertainty.

\section{Comfort varies based on perceived benefits}

Perceived benefits of car-based recording impacted perceived comfort. Respondents perceived improved driving for individuals in vehicles with recording present, public safety benefits associated with having cameras present in more locations, and general improved road safety. However, perceived benefits varied for hypothetical drivers versus bystanders.

\section{Perceived benefits impacted comfort with recording}

Study 2 participants' comfort tended to increase when purpose and benefits of recording were clear, especially when they felt data was going to be used for safety. P10 provides an example of this dynamic (Figure 3). Participants tended to be less comfortable when benefits were unclear; P03 explained, "Maybe in a big city center, it makes sense. But where I'm living now, so quiet place. I don't need to be recorded or provide information or to receive any information."

P10 (F, Swiss): Benefit-driven comfort

Activities: Drink in café, biking, walking, swimming Baseline perceived recording:

- Knew about CCTV camera at the campus where she worked, and is comfortable, because it is for safety

- Believed she walked past houses/shops with security cameras; less comfortable, because purpose is unclear

Comfort with car-based recording:

- Comfortable when biking, because it might make her safer

- Otherwise did not "see much use to it"

P11 (M, US): Public/private space-driven comfort

Activities: Leisure around house (e.g., sitting on porch, playing with kids in the yard), biking, driving

Baseline perceived recording:

- Minimal: GPS, computer network tracking

- Slightly uncomfortable, but "I don't know why"

Comfort with car-based recording:

- Comfortable in public spaces, for example on the road, because "there's a shared responsibility"

- Less comfortable at home, because it is private property

- Less comfortable when with kids, even in public

Figure 3. Sample Study 2 participants

Study 1 respondents described activities they performed regularly that they felt would benefit from car-based recording as well as the situations (events) they felt would most benefit from car-based recording in the past year (Table 2). Overall, over half of respondents described driving-related activities they felt would benefit from recording $(62 \%)$. These related to helping them drive by improving their reaction times, helping them avoid traffic, providing directions, or helping with parking. Similarly, some respondents described the potential for car-based recording to create a record of incidents for insurance or legal purposes (29), for example, "If I were to get in an accident with one of the many people who cut me off maybe it would capture it and help with the insurance claim."

For specific events, respondents also tended to feel they would have benefited from having accidents recorded (22\% overall) or having recording present during near-accidents or other incidents of bad driving (25\%). They tended to feel recording features might have prevented some incidents, for example, "I was rear ended on the interstate, it would have been nice if the other car had sensors that would have prevented that" $(D N)$ or could have caught the drivers responsi- 


\begin{tabular}{|c|c|c|c|c|c|}
\hline \multicolumn{6}{|l|}{ Activities } \\
\hline & Examples & Driver & Drive near & Bystander & Total \\
\hline Help one drive & $\begin{array}{l}\text { improve reactions, provide directions, help } \\
\text { with traffic }\end{array}$ & $112(80 \%)$ & $56(64 \%)$ & $49(40 \%)$ & $217(62 \%)$ \\
\hline Help others/catch others & help/catch bad drivers & $13(9 \%)$ & $14(16 \%)$ & $37(30 \%)$ & $64(18 \%)$ \\
\hline Promote public safety & protect pedestrians/bikers, deter crime & $8(6 \%)$ & $2(2 \%)$ & $23(19 \%)$ & $33(9 \%)$ \\
\hline Create a record & record for legal or insurance purposes & $8(6 \%)$ & $10(11 \%)$ & $11(9 \%)$ & $29(8 \%)$ \\
\hline None/unclear/other & - & $6(4 \%)$ & $7(8 \%)$ & $10(8 \%)$ & $23(7 \%)$ \\
\hline \multicolumn{6}{|l|}{ Events } \\
\hline & Examples & Driver & Drive near & Bystander & Total \\
\hline Bad-driving incident & $\begin{array}{l}\text { drove badly, saw bad driving, near- } \\
\text { accident }\end{array}$ & $29(21 \%)$ & $34(39 \%)$ & $24(20 \%)$ & $87(25 \%)$ \\
\hline Accident & accident/driving-related incident occurred & $29(21 \%)$ & $18(21 \%)$ & $29(24 \%)$ & $76(22 \%)$ \\
\hline Wanted directions/traffic aid & needed directions, was lost, stuck in traffic & $47(34 \%)$ & $14(16 \%)$ & $14(11 \%)$ & $75(21 \%)$ \\
\hline Crime/felt unsafe & non-driving-related crime, felt unsafe & $2(1 \%)$ & $4(5 \%)$ & $19(16 \%)$ & $25(7 \%)$ \\
\hline Help driving in spec. situation & help in a storm, with parking, etc. & $12(9 \%)$ & 0 & $13(11 \%)$ & $25(7 \%)$ \\
\hline Good event & record of positive event (drove well, view) & $6(4 \%)$ & $4(5 \%)$ & $8(7 \%)$ & $18(5 \%$ \\
\hline None/unclear/other & - & $15(11 \%)$ & $13(15 \%)$ & $15(12 \%)$ & $43(12 \%)$ \\
\hline
\end{tabular}

Table 2. Study 1: Activities and events participants would want recorded by car-based recording/sensing, by condition. Some items were double coded.

ble. They also felt they could have benefited from GPS when stuck in traffic or in need of directions.

Similarly, some Study 2 participants felt that "smart"-carbased recording might hold others accountable for poor driving or improve general traffic conditions, for example, "help trains to understand if there are any delays ahead” (P12).

In Study 1, perceptions of the benefits of "smart"-car-based recording varied by stakeholder condition. Respondents in the bystander condition were less likely to describe activities related to helping them drive $(80 \%$ for driver, $64 \%$ for drive near, $40 \%$ for bystander) or driving-related events. Frequencies were significantly lower for driving-related activities and events in the bystander versus driving conditions (Chi Squared tests, $p<0.01$ for all), except for events in the drive near condition (lower, but not significantly, $p=0.15$ ).

Instead of driving-related benefits, respondents in the bystander condition tended to describe benefits related to promoting general public safety (19\% of bystander respondents), including protecting pedestrians and bicyclists, providing public health services, or deterring crime, "If I were in an accident, it would be helpful for a car to call an ambulance automatically, in case I am unconscious" (B), or "Crossing the street would likely be safer. I wouldnt have to worry about getting hit by a distracted driver" (B). They also tended to describe the potential to benefit from car-based recording during events related to crime or feeling unsafe, for example "Someone shot out the window in my fathers car with a pellet ri$f l e "(B)$. Or, they described the potential for the features to help or catch others (30\%), focused on helping others' driving or catching others when they drove badly or broke the law. Some Study 2 respondents also described general safetyrelated benefits in bystander scenarios. They described protection from cars when biking or crime prevention, for exam- ple: "if someone begins to attack or vandalize their car, that car would be... recognizing this has happened" (P15).

\section{Mixed perception of legal uses}

There were mixed attitudes toward legal use of data recorded by "smart" cars. Although many Study 1 respondents wanted others' poor driving captured, about half did not want their own bad or illegal driving recorded (Table 3). Respondents described not wanting their behaviors like running stop signs, speeding, or phone use and texting captured, reflecting a fear that "it might see me texting while driving, which $i$ should not" (DN). Study 2 respondents also described fears that data might be used against them, for example, "if you're guilty, like, you don't want that data” (P09).

\section{Car-based recording and semi-private spaces}

Car-based recording can include publicly visible spaces that may be considered private for reasons including the performance of personal or potentially embarrassing activities, the presence of children, and proximity to home. This can create a dynamic of semi-private spaces in and around cars.

\section{Sensitive activities in and near cars}

Choe et al. found a "long tail" of sensitive activities people would not want recorded in the home [8]. Study 1 respondents described a similar range of potentially sensitive activities and events in and around cars.

Although the interior of cars on the road are typically visible, respondents described personal or potentially embarrassing activities they performed in their cars that they would not want recorded. These included contentious acts like road rage: "...cussing other drivers out and at times being racially insensitive” (DN) or general anger: “...yelling at my daughter and making her so upset that she began to cry" $(B)$. Some respondents also described talking or singing in their cars. 


\begin{tabular}{l|l|lll|l}
\hline & Examples & Driver & Drive near & Bystander & Total \\
\hline Bad/illegal driving & phone use, running lights, speeding & $86(61 \%)$ & $51(59 \%)$ & $37(30 \%)$ & $174(50 \%)$ \\
Oral expression/media use* & singing, radio, conversations & $9(6 \%)$ & $12(14 \%)$ & $5(4 \%)$ & $26(7 \%)$ \\
Location tracking & unease w/location/schedule being known & $12(9 \%)$ & $6(7 \%)$ & $7(6 \%)$ & $25(7 \%)$ \\
Contentious acts* & road rage, disciplining, angry acts & $13(9 \%)$ & $5(6 \%)$ & $5(4 \%)$ & $23(7 \%)$ \\
Social. awkward acts* & picking one's nose, vomiting & $4(3 \%)$ & $5(6 \%)$ & $7(6 \%)$ & $16(5 \%)$ \\
Gen. monitoring & unease w/tracking or info being collected & $4(3 \%)$ & $3(3 \%)$ & $12(10 \%)$ & $19(5 \%)$ \\
Self-appearance/Pers. hygiene* & nudity, partial dress, grooming & $3(2 \%)$ & $1(1 \%)$ & $10(8 \%)$ & $14(4 \%)$ \\
Alcohol or tobacco* & smoking, drinking, going to a bar & $2(1 \%)$ & $2(2 \%)$ & $6(5 \%)$ & $10(3 \%)$ \\
Illegal activities (non-driving) & illegal drugs, littering & $2(1 \%)$ & $4(5 \%)$ & $4(3 \%)$ & $10(3 \%)$ \\
Activities around the house & going out to the car, mowing the lawn, & 0 & 0 & $8(7 \%)$ & $8(2 \%)$ \\
& standing in living room & $3(2 \%)$ & $1(1 \%)$ & $3(2 \%)$ & $7(2 \%)$ \\
Eating* & eating food & 0 & 0 & $6(5 \%)$ & $6(2 \%)$ \\
Pet activities & walking the dog, picking up dog poop & $2(1 \%)$ & $1(1 \%)$ & $2(2 \%)$ & $5(1 \%)$ \\
Intimacy* & sexual acts, kissing & 0 & 0 & $4(3 \%)$ & $4(1 \%)$ \\
Walking & walking outside & 0 & 0 & $3(2 \%)$ & $3(1 \%)$ \\
Physical activity* & exercising, dancing & $16(11 \%)$ & $2(2 \%)$ & $10(8 \%)$ & $28(8 \%)$ \\
None/unclear/other & - & &
\end{tabular}

Table 3. Study 1: activities participants would not want recorded by car-based recording/sensing, by condition. (*) indicates category based on Choe et al. [8]. Some items were double coded.

Other respondents did not want potentially embarrassing socially awkward acts like picking one's nose or varied selfappearance/personal hygiene-related behaviors including nudity or partial dress in, or visible from, cars recorded: "Probably me walking out into my driveway in my underwear to get something out of the car" $(B)$. Several respondents described alcohol or tobacco use or non-driving-related illegal activities like drug use. Others described intimate acts, for example, "I one time had sex on my patio with my girlfriend, and would not want cars recording that" $(B)$.

Some activities were less obviously embarrassing and reflected the use of cars and areas nearby as personal spaces. For example, some respondents did not want to be recorded eating in their cars. Others did not want to be recorded walking outside, or while "Picking up my dogs poop" (B). Respondents also found physical activity potentially sensitive, for example, "Going running, where I would get all sweaty and gross..." (B), or did not want to be recorded during everyday activities in and around their homes, such as: "Standing in my living room" (B).

\section{Mental models of privacy levels}

In line with those varied sensitive activities, some Study 2 participants perceived comfort with "smart"-car recording based on a space's perceived privacy level. Perceptions of privacy tended to depend on mental models of public and private spaces and activities. These included perceived legal boundaries, leisure versus utilitarian activities, ability to control privacy, and the presence or absence of children.

Some participants relied on perceptions of law to differentiate privacy levels. Several described private property, including their homes or college campuses, as being more private, and were less comfortable with recording in those locations. Alternatively, some participants considered spaces like roads to be public because it is legal to take pictures in the street. P11 provides an example (Figure 3).

Other participants distinguished privacy levels based on activities. For some, privacy level varied based on whether they were performing leisure or utilitarian activities, for example: "then I am in a place in which I went to enjoy myself and to be-it's like the public and the private are at least divided in that way" (P01). Alternatively, some participants felt that specific activities were embarrassing and did not want them recorded. For example P07 did not want to be recorded on her break because she does not "really like the fact that I'm stuck working at Dunkin' Doughnuts.”

Perceptions of comfort also varied based on the presence of children. For example, P12 works at an elementary school. She was always uncomfortable with recording when the students were present at the school because: "I work with elementary aged school kids, and we go to great lengths to, you know, make sure that their privacy is protected."

P05, alternatively, distinguished public and private spaces based on her ability to control her privacy:

[in the home] if you want privacy, you draw the curtains. And you accept that if you're doing something sort of in the living room on the ground floor then anybody who drives by can see what you're doing...So you don't have quite the same expectation or concern for privacy there...A doctor's office, to me, is different because - you've got no choice about where you go in or come come back out.

\section{Perceptions of collection, access, identifiability}

Participants tended to also perceive comfort as depending on level of data collection, access, and perceived identifiability. 
Some Study 2 participants were concerned about how much data might be collected. P04 explained, "they would actually get my every step, what I'm actually doing. Because I will be passing smart cars, passing by the smart cars. And they will, they can monitor my entire day." Others were concerned about data being retained for long periods of time, or about opportunities for re-use or malicious interception. P08 explained: "someone may be able to hack the system in a way that may be able to steal the information."

Comfort also varied with potential level of data collection. For example, several participants were more comfortable with car-based radar than external cameras, because "Somehow cars reading information about other cars seems...seems less personal" (P05). Some participants' comfort also varied based on how identifiable they felt during activities. These feelings of identifiability often did not relate to technical anonymity, but, rather, how much they felt they could be recorded as an individual. For example, P15 felt he was more identifiable as a pedestrian than as a driver because more of his body was visible. Similarly, P02 was less comfortable when he felt someone could "actually see me driving" (P02).

Vehicle-based recording is still relatively new, so there may be increased uncertainty about how the collection is performed, who has access to data, how it is stored, and level of identifiability. This dynamic could prompt discomfort.

\section{Need for trusted, usable privacy-preserving mechanisms} We asked Study 2 participants about three privacy-preserving techniques: storing data locally in the car for only diagnostic and driving purposes (local storage), blurring personally identifiable information like faces and license plates (PII blurring), and being able to set locations to private for which no data would be sent to a service provider (geo-fencing).

\section{Mechanisms tended to be perceived positively}

Participants tended to perceive the mechanisms positively. They tended to feel such mechanisms would increase transparency around data access and flow, increase control, and decrease identifiability. Participants tended to feel that local storage, or local storage with geo-fenced boundaries, could reduce potential for undesired data flow, for example to malicious entities or for undesired commercial use. P13 described: "in general I would be more comfortable with this type of data stored and processed locally than going off God knows where and then being used for whatever..." Similarly, participants generally liked the idea of increased control from geofencing.

Participants also felt PII blurring would decrease identifiability. However, some felt this comfort was specific to locations with many people, for example: "there are so many other people in the shopping center, at the cinema, that it will be really hard to recognize you" (P09).

\section{Need for trust and usability}

Despite generally increased comfort, some participants distrusted implementation of such mechanisms. Some participants felt that data, once captured could still be shared. They worried about ownership of locally stored data, including a car owner accessing the data, as well as security breaches, explaining: “...as long as it's recorded, then they could always have a failure" (P04).

Similarly, some participants distrusted face and license plate blurring to provide adequate privacy protection. Several participants had mental models of blurring that reflected an ability to undo the protection, explaining: "if I take a picture and I put it into Photoshop and I blur the face or something I still have the original file, picture, and it doesn't mean a thing" (P04). Others felt that features other than a face and license plate can make someone identifiable, for example, "I think there's identifiable information everywhere from the color of your home and the clothes you're wearing" (P11).

Participants also expressed concern about the usability of geo-fencing. Geo-fencing requires a usable system to create access-control boundaries. P14 pointed out that a user might have to sign up for the recording service to use it: “...it seems like there might be some - some issues with that actually working for people who weren't already part of the - of the data collecting - like, the program..."

Participants described a range of locations they might want to set private with such a system, including home, work, areas near home, friends' or relatives' homes, schools, and doctors' offices. A few participants also described activities or events they would want to set private, for example, "anytime that you are drinking” (P01). However, a few participants explained that they did not feel they had control over certain areas or felt certain areas were public, for example: "[the shopping center] - these are places with a lot of people and it's actually impossible to set these places as private" (P09).

Thus, effective privacy-protective mechanisms would need to address clearly-defined risk models, for example levels of identifiability, as well as be transparently explained, include usably-provided desired levels of control, and, where possible, include guarantees around protections.

\section{DISCUSSION}

Vehicles may bring recording into unexpected places, which may impact varied stakeholders whose comfort relies on different perceived benefits. This comfort also depends on perceptions of semi-private spaces, creating a complex dynamic for assessing privacy levels. There are a variety of areas for future work to help guide the development of vehicle-based recording that is sensitive to these general perceptions.

\section{Notification and awareness for vehicle-based recording}

Vehicle-based recording can occur in surprising moments. Iachello et al. describe the challenge of "lessening the cultural stress that many indicate today as an acceptance challenge of ubicomp and facilitating the process of 'shifting the technology the background"' [13]. Vehicles are already a common technology. Facilitation must, therefore, focus on the acceptance challenge of shifting perceptions to vehicles as potential ubiquitous recording devices.

This creates a challenging dynamic. Respondents often felt unrecorded when they could have been recorded by vehicles, and typically did not have a clear idea of how to recognize 
cars with recording capabilities. The emergent aspects of vehicle-based recording also add uncertainty around data collection and access. This may create a "chilling" effect. Most cars do not currently include recording, but any car may include recording. Thus, people may modify their behaviors in anticipation of recording that may or may not be present.

This awareness gap may close as vehicle-based recording becomes more common, and mental models adjust. However, in the near-term, awareness could be increased through user education or vehicle-based notifications. Future work could examine measures for creating effective notifications for phasing in vehicle-based recording, or more broadly, any technology that may add recording in a currently unperceived manner. Such notifications would need to address issues such as balancing increased awareness with risk perceptions.

\section{Designing for varied perceptions}

There are also opportunities to design vehicle-based technologies in ways that account for the varied perceptions seen in our studies.

\section{Designing for varied stakeholders}

Participants perceived the benefits of recording as important. However, perceived benefits varied for hypothetical bystanders versus drivers. While designers may focus on creating features for drivers' benefits, including features that also emphasize benefits for bystanders may increase general acceptance. Such benefits could address public safety issues, including bicyclist or pedestrian safety.

\section{Considering varied sensitivities}

Vehicle-based recording may enter semi-private spaces, which are visible to the public but perceived as private. Vehicles may move in and out of such spaces, and different people may have different mental models for considering spaces private. Data access and use policies should consider the full, and potentially complex, range of data that might be captured by vehicle-based recording that people may consider sensitive. Vehicles may capture activities that may appear innocuous but that people may consider to be highly personal, ranging from mowing the lawn to picking one's nose in a car.

\section{Trustworthy and usable privacy preservation}

Participant attitudes toward privacy-preserving mechanisms were positive but such mechanisms would require usable controls and trustworthy guarantees of protection. Mechanisms would need to capture complex, varied sensitivities, with usable controls. Creating controls, like geo-fences that captured that complexity would be challenging and require additional work. We saw, for example, that participants wanted areas kept private over which they did not feel they had direct control. Additionally, some respondents wanted activity-based controls, while others wanted location-based controls. An effective system that allowed bystanders to control their privacy for vehicle-based recording might include controls that combined a number of factors, including location, time, and allowing individuals to opt out [4].
Respondents also distrusted some privacy-preservation aspects of the mechanisms. They felt blurring could be "undone" or local storage hacked. Effective mechanisms might need guarantees of protections, where possible. For example, blurring could provide a feedback mechanism, or local storage could provide physical means of blocking transmission or protecting the storage device.

\section{Additional factors to explore}

We focused on eliciting general reactions rather than on how people trade off risks and benefits. However, tradeoffs emerged around using car-based recording for legal purposes, especially for capturing one's own poor and illegal driving versus capturing others' poor and illegal driving. Follow-up work could more deeply address how people would trade off the benefits of catching others' poor behaviors versus the potential of being caught oneself.

We also grounded our studies in attitudinal reactions to past events so we could perform more general exploratory work with limited hypothetical reactions. Future work could focus on examining reactions to car-based recording in-situ either through the use of focused experience sampling, or through reactions to recording. For example, work could ask participants to react to potential sensing as they went about daily activities, ask participants to provide feedback to data collected by a vehicle, or ask participants to react to, or use, different privacy-preserving techniques for actual data.

There are also many factors that may impact comfort that we did not see, and that might emerge in larger, or more focused, samples. We specifically hoped to see factors that might arise from differences in US and Swiss privacy culture and policy environments. These differences might emerge in a larger study. Other factors that contribute to a potential benefit or risk perception profile might also contribute interesting differences, such as urban or rural residents, types of transportation typically used, and broad attitudinal factors.

\section{CONCLUSION}

Vehicle-based recording adds dynamics to existing ubiquitous recording and sensing. It brings increasing recording and sensing into varied spaces that can be perceived as semiprivate, and comfort with such recording can vary based on car-specific benefits. As an emerging technology it also presents uncertainty around collection, access, and identifiability. However, as vehicle-based recording technologies are developed there are opportunities to create features and user education that are sensitive to public perceptions.

\section{ACKNOWLEDGMENTS}

We would like to thank Martin Ortlieb, Ulfar Erlingsson, Ed Chi, Patri Friedman, Kerwell Liao, and Jeff Tamer, as well the anonymous reviewers for their valuable contributions to this work.

We would also like to dedicate this paper in memory of Dan Fredinburg, whose passion for privacy inspired us to believe in the impossible. 


\section{REFERENCES}

1. Privacy and security: For Street View. http://www.google.com/maps/about/behind-thescenes/streetview/privacy/\#streetview.

2. Onstar services. https://www.onstar.com/us/en/services/services.html, 2014.

3. Abowd, G. D., Hayes, G. R., Iachello, G., Kientz, J. A., Patel, S. N., Stevens, M. M., and Truong, K. N. Prototypes and paratypes: Designing mobile and ubiquitous computing applications. Pervasive Computing, IEEE 4, 4 (2005), 67-73.

4. Barhm, M. S., Qwasmi, N., Qureshi, F. Z., and El-Khatib, K. Negotiating privacy preferences in video surveillance systems. In Modern Approaches in Applied Intelligence. Springer, 2011, 511-521.

5. Berinsky, A. J., Huber, G. A., and Lenz, G. S. Evaluating online labor markets for experimental research: Amazon.com's Mechanical Turk. Political Analysis 20, 3 (2012), 351-368.

6. Billson, J. M. Conducting focus group research across cultures: Consistency and comparability. UK: ESRC Research Group on Wellbeing in Developing Countries (2006).

7. Buhrmester, M., Kwang, T., and Gosling, S. D. Amazon's Mechanical Turk a new source of inexpensive, yet high-quality, data? Perspectives on Psychological Science 6, 1 (2011), 3-5.

8. Choe, E. K., Consolvo, S., Jung, J., Harrison, B., and Kientz, J. A. Living in a glass house: A survey of private moments in the home. In Proceedings of the 2011 ACM Conference on Ubiquitous Computing, ACM (2011), 41-44.

9. Choe, E. K., Consolvo, S., Jung, J., Harrison, B., Patel, S. N., and Kientz, J. A. Investigating receptiveness to sensing and inference in the home using sensor proxies. In Proceedings of the 2012 ACM Conference on Ubiquitous Computing, ACM (2012), 61-70.

10. Consolvo, S., and Walker, M. Using the experience sampling method to evaluate ubicomp applications. IEEE Pervasive Computing, 2 (2003), 24-31.

11. Denning, T., Dehlawi, Z., and Kohno, T. In situ with bystanders of augmented reality glasses: Perspectives on recording and privacy-mediating technologies. In Proceedings of the 2014 ACM Conference on Human Factors in Computing Systems, ACM (2014), 2377-2386.

12. Friedman, B., Kahn Jr, P. H., Hagman, J., Severson, R. L., and Gill, B. The watcher and the watched: Social judgments about privacy in a public place. Human-Computer Interaction 21, 2 (2006), 235-272.

13. Iachello, G., Truong, K. N., Abowd, G. D., Hayes, G. R., and Stevens, M. Prototyping and sampling experience to evaluate ubiquitous computing privacy in the real world.
In Proceedings of the SIGCHI conference on Human Factors in computing systems, ACM (2006), 1009-1018.

14. Ion, I., Sachdeva, N., Kumaraguru, P., and Čapkun, S. Home is safer than the cloud!: Privacy concerns for consumer cloud storage. In Proceedings of the Seventh Symposium on Usable Privacy and Security, ACM (2011), 13.

15. Ipeirotis, P. Turker demographics vs internet demographics, 2009.

16. Kessler, A. M., and Vlasic, B. Semiautonomous driving arrives, feature by feature. New York Times, April 2015.

17. Massimi, M., Truong, K. N., Dearman, D., and Hayes, G. R. Understanding recording technologies in everyday life. Pervasive Computing, IEEE 9, 3 (2010), 64-71.

18. Mennicken, S., and Huang, E. M. Hacking the natural habitat: An in-the-wild study of smart homes, their development, and the people who live in them. In Pervasive Computing. Springer, 2012, 143-160.

19. NASA. Prepping advanced driver assistance for mainstream vehicles. NASA Tech Briefs, September 2014.

20. Neubecker, R. Lower your car insurance bill, at the price of some privacy. New York Times, August 2014.

21. Nguyen, D. H., Bedford, A., Bretana, A. G., and Hayes, G. R. Situating the concern for information privacy through an empirical study of responses to video recording. In Proceedings of the 2011 ACM Conference on Human Factors in Computing Systems, ACM (2011), 3207-3216.

22. Nguyen, D. H., Marcu, G., Hayes, G. R., Truong, K. N., Scott, J., Langheinrich, M., and Roduner, C. Encountering SenseCam: Personal recording technologies in everyday life. In Proceedings of the 2009 ACM Conference on Ubiquitous Computing, ACM (2009), 165-174.

23. Norberg, P. A., Horne, D. R., and Horne, D. A. The privacy paradox: Personal information disclosure intentions versus behaviors. Journal of Consumer Affairs 41, 1 (2007), 100-126.

24. Oulasvirta, A., Pihlajamaa, A., Perki, J., Ray, D., Vhkangas, T., Hasu, T., Vainio, N., and Myllymki, P. Long-term effects of ubiquitous surveillance in the home. In Proceedings of the 2012 ACM Conference on Ubiquitous Computing, ACM (2012), 41-50.

25. Pickler, N. 'Smart car' tech encouraged in U.S. ABC News, July 2014.

26. Procyk, J., Neustaedter, C., Pang, C., Tang, A., and Judge, T. K. Exploring video streaming in public settings: Shared geocaching over distance using mobile video chat. In Proceedings of the 2014 ACM Conference on Human Factors in Computing Systems, ACM (2014), 2163-2172. 
27. Schnorf, S., Sedley, A., Ortlieb, M., and Woodruff, A. A comparison of six sample providers regarding online privacy benchmarks. In SOUPS Workshop on Privacy Personas and Segmentation (2014).

28. Thomaz, E., Parnami, A., Bidwell, J., Essa, I., and Abowd, G. D. Technological approaches for addressing privacy concerns when recognizing eating behaviors with wearable cameras. In Proceedings of the 2013 ACM Joint Conference on Pervasive and Ubiquitous Computing, ACM (2013), 739-748.

29. Thomaz, E., Parnami, A., Bidwell, J., Essa, I., and Abowd, G. D. Technological approaches for addressing privacy concerns when recognizing eating behaviors with wearable cameras. In Proceedings of the 2013
ACM Joint Conference on Pervasive and Ubiquitous Computing, ACM (2013), 739-748.

30. Trop, J. A black box for car crashes. New York Times, July 2013.

31. Tuttle, B. Big data is my copilot: Auto insureres push devices that track driving habits. Time, August 2013.

32. Vanderhulst, G., Van den Broeck, M., and Kawsar, F. Exploring the design space for geo-fenced connected devices and services at home. In Proceedings of the 2014 ACM International Joint Conference on Pervasive and Ubiquitous Computing: Adjunct Publication, ACM (2014), 1321-1327. 